Limnetica, 26 (2): 351-358 (2007). DOI: 10.23818/limn.26.30

(c) Asociación Ibérica de Limnología, Madrid. Spain. ISSN: 0213-8409

\title{
Diatomeas y calidad del agua de los ríos del Macizo Central Gallego (Ourense, N.O. España) mediante la aplicación de índices diatomológicos
}

\author{
María Penalta-Rodríguez y Ma Carmen López-Rodríguez
}

Departamento de Botánica. Facultad de Biología. Universidad de Santiago de Compostela. Campus sur. 15071. Santiago de Compostela. A Coruña. España

* Corresponding author: bvmariap@usc.es

\begin{abstract}
Diatoms and water quality of the rivers of the Galician Central Massif (Ourense, N.O. Spain) through the use of diatom indices

Mountain rivers diatoms from a complex of sierras that form the Galician Central Massif (Ourense province), place of communitarian importance, have been studied. For that study, eight rivers and one spring have been sampled, over one and a half years (from 2001 to 2003) and water samples were also taken to obtain some physico-chemical parameters (pH, conductivity, temperature, and dissolved oxygen). The most abundant species are Achnanthidium minutissimum, Diatoma mesodon, Fragilaria capucina, Gomphonema parvulum, G. rhombicum, and Hannaea arcus.

With the diatoms' abundances, the IPS, IBD, and CEE indices were calculated to study the water quality, using the OMNIDIA program. The results of the indices classify the waters of these mountain rivers as of good or very good quality.
\end{abstract}

Key words: Diatoms, Ourense, Central Massif, water quality, IPS, IBD, CEE, OMNIDIA.

\section{RESUMEN}

Diatomeas y calidad del agua de los ríos del Macizo Central Gallego (Ourense, N.O. España) mediante la aplicación de índices diatomológicos

Se han estudiado diatomeas de ríos de montaña de un conjunto de sierras que constituyen el Macizo Central Gallego (provincia de Ourense), lugar de importancia comunitaria (LIC). Se han muestreado 8 ríos y 1 fuente para dicho estudio durante un período de año y medio (2001-2003) y también se han tomado muestras del agua para la obtención de algunos parámetros físico-químicos ( $\mathrm{pH}$, conductividad, temperatura y oxígeno disuelto). Las especies más abundantes fueron Achnanthidium minutissimum, Diatoma mesodon, Fragilaria capucina, Gomphonema parvulum, G. rhombicum y Hannaea arcus.

Con las abundancias de las diatomeas se calcularon los índices IPS, IBD y CEE para el estudio de la calidad de las aguas, utilizando el programa OMNIDIA. Los resultados de los índices clasifican las aguas de estos ríos de montaña como de calidad buena o muy buena.

Palabras clave: Diatomeas, Ourense, Macizo Central Gallego, calidad del agua, IPS, IBD, CEE, OMNIDIA.

\section{INTRODUCCIÓN}

El Macizo Central Gallego (MCG), lugar de interés comunitario en la reserva de espacios naturales europeos "Natura 2.000", es una formación montañosa situada en el noroeste de la Península
Ibérica e incluye tres cadenas montañosas: Manzaneda, Chandrexa de Queixa e Invernadeiro.

Los trabajos ficológicos en esta zona de la comunidad gallega son escasos. Noguerol (1993) estudió la flora algal únicamente en la Sierra de Invernadeiro y López \& Penalta (2004) 
y Penalta \& López (2006) en ríos y fuentes de todo el MCG. En el resto de Galicia destacan los trabajos taxonómicos de Gamundi (1911) y Varela $(1976,1982,1991)$ referidos a diatomeas. Los trabajos de Margalef $(1955,1956)$ estudian todos los grupos taxonómicos de la flora dulceacuícola, incluidas las diatomeas, y son las publicaciones más exhaustivas existentes hasta el momento en Galicia.

Los trabajos de Ector (1992) y De la Peña (2003) inician la utilización de índices diatómicos para valorar la calidad de aguas corrientes continentales en las estaciones de aforo de la Confederación Galicia-Costa y en cuatro ríos de la provincia de A Coruña, respectivamente.

Los objetivos de este estudio han sido en primer lugar, el estudio detallado de las diatomeas del Macizo Central Gallego, para incrementar el conocimiento de la biodiversidad ficológica de las aguas continentales en Galicia. $\mathrm{Y}$, en segundo lugar se ha utilizado a este grupo de algas, para el cálculo de los índices diatómicos IPS, IBD y CEE, con el objeto de valorar la calidad de las aguas de estos ríos de la montaña gallega, hasta ahora nunca estudiada.

\section{MATERIAL Y MÉTODOS}

\section{Área de estudio}

El Macizo Central Gallego (Fig. 1, Tabla 1) está situado al sureste de la provincia de Ourense (Galicia), tiene una extensión de 46983 ha y una altitud máxima de $1778 \mathrm{~m}$, siendo la segunda formación montañosa más alta de Galicia. Aquí se encuentran numerosos ríos de pequeño tamaño que acaban vertiendo sus aguas en afluentes del Miño. La mayoría poseen una vegetación de ribera típica (Salix atrocinerea, Betula alba, Fraxinus angustifolia, Alnus glutinosa y Corylus avellana).

Desde el punto de vista biogeográfico, el MCG se sitúa entre los límites de la región Eurosiberiana y Mediterránea. El clima es templado-fresco (Carballeira et al., 1983).

En cuanto a su geología, en la zona sur del Macizo dominan pizarras del Ordovícico medio y Silúrico inferior y en la parte noreste predomina

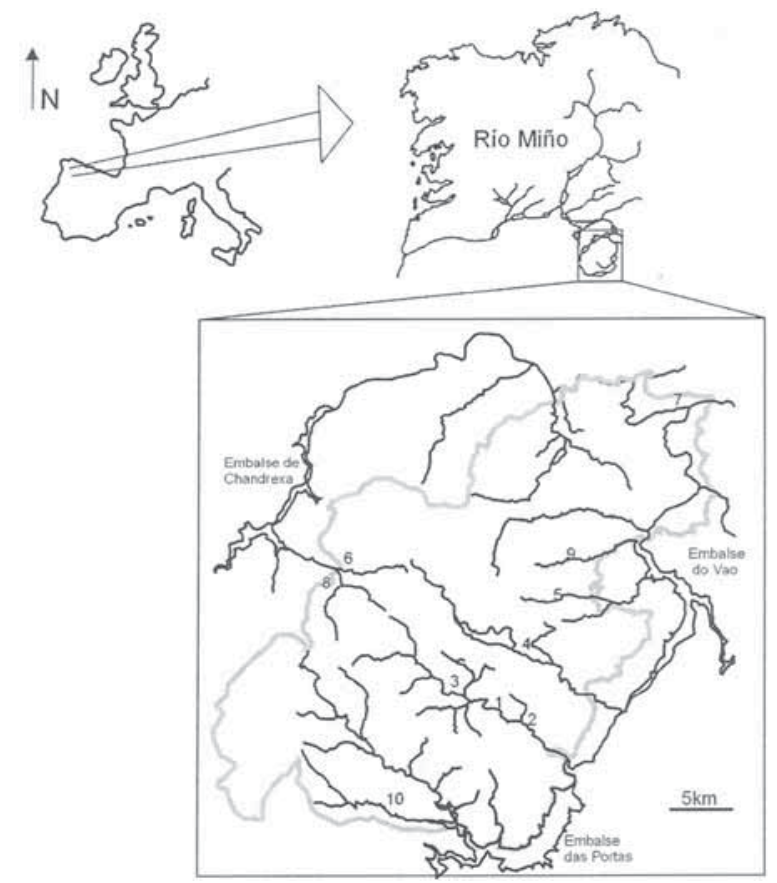

Figura 1. Localización de los ríos estudiados y de las estaciones muestreadas. Location of the studied rivers and the sampled stations.

una intrusión granítica datada del Ordovícico superior (I.G.M.E., 1977).

\section{Metodología}

Las muestras se tomaron entre los años 20012003 , en 8 ríos y una fuente (Tabla 1). Las diatomeas fueron recogidas según el protocolo europeo (Kelly et al., 1998; European Commite of Standardization, 2002) cepillando en cada localidad la superficie de 5 piedras sumergidas, de $10-20 \mathrm{~cm}$ de diámetro, que fueron obtenidas en zonas del río bien iluminadas y con corriente. Las muestras se conservaron en formaldehído al $4 \%$. En el laboratorio, la materia orgánica se eliminó mediante la oxidación con peróxido de hidrógeno al $40 \%$ (Prygiel \& Coste, 2000). Los frústulos se limpiaron con agua destilada, se deshidrataron sobre cubreobjetos redondeados y se montaron en Naphrax $®$.

Para el recuento e identificación de las diatomeas se utilizó un microscopio OLYMPUS BX61 con contraste interferencial Nomarski y 
Tabla 1. Localidades estudiadas y tipo de sustrato (ar: arena, gv: grava, ca: canto, ro: roca). Studied locations and substrate type. (ar: sand, gv: gravel, ca: pebble, ro: rock)

\begin{tabular}{clcccccc}
\hline Código & Localidad & Coordenadas & Fecha & longitud km & $\begin{array}{c}\text { anchura } \\
\text { media }\end{array}$ & $\begin{array}{c}\text { tipo de } \\
\text { substrato }\end{array}$ & $\begin{array}{c}\text { sustrato } \\
\text { cuenca }\end{array}$ \\
\hline 1 & Conso 1 & 29T6441504669700 & 08 -dic-01 & 13.6 & 2 & ar, ca & Metamórfico \\
2 & Conso 2 & 29T6464844667129 & 17 -jun-03 & 13.6 & 9.8 & ca, gr & Metamórfico \\
3 & Pradoalbar & 29T6417884670533 & 17 -jun-03 & 4.7 & 7 & ro, ca, gr & Metamórfico \\
4 & Cenza & 29T6472504671662 & 23 -abr-02 & 15.9 & 3 & ro, ca, & Sedimentario \\
5 & Cernado & 29T6499704678423 & $17-$ jun-03 & 6.3 & 6 & gra, ar & Sedimentario \\
6 & Palleiras & 29T6361924681500 & 18 -jun-03 & 6.7 & 5.5 & ar, ca & Sedimentario \\
7 & Caneiro & 29T6473984687699 & $17-$ jun-03 & 22.9 & 11.5 & ar, ca & Granítico \\
8 & Tornos & 29T6364324683447 & 18 -jun-03 & 2.8 & 3.5 & ro, ca & Granítico \\
9 & Grixoa & 29T6510664676253 & 17 -jun-03 & 7.6 & 6.5 & gr, ar & Granítico \\
10 & Fonte Ribeira Pequena & 29T6377254664100 & 05-nov-02 & - & - & - & - \\
\hline
\end{tabular}

se contaron unas 400 valvas, estimándose la abundancia relativa de cada taxón en la muestra.

Para la identificación de los taxones se siguieron las siguientes referencias bibliográficas: Hustedt (1930); Bourrelly (1968); Germain (1981); Krammer et Lange-Bertalot (1986, 1988, 1991a, b); Lange-Bertalot et Krammer (1989); Krammer (2000, 2002, 2003); Lange-Bertalot (2001) y Aboal et al. (2003).

Se calcularon tres índices de diatomeas para valorar la calidad del agua: IPS, IBD y CEE, mediante el software OMNIDIA 4.1. Se han seleccionado estos tres índices por ser los más generales y los que dan mejores resultados en relación con los parámetros físico-químicos para Europa y para Galicia (Ector, 1992; Prygiel et al, 1999; De la Peña, 2003). Los valores obtenidos del cálculo de estos índices definen cinco clases de calidad que son las siguientes; mala $(<5)$, deficiente (5-9), aceptable (9-13), buena (13-17) y muy buena ( $>17)$.

También se tomaron medidas "in situ" del $\mathrm{pH}$, conductividad, oxígeno disuelto y temperatura, utilizando un pHmetro HANNA HI 9024C, un conductivímetro HANNA HI 9033 y un oxímetro CRISON 45.

\section{RESULTADOS}

Las variables físico-químicas medidas en los ríos estudiados nos indican (Tabla 2) que son aguas de carácter ligeramente ácidas a neutras variando el pH entre 6.3 y 7.6. La conductividad es muy baja,

Tabla 2. Variables físicas y químicas medidas en cada estación. Physical and chemical variables measured at each station.

\begin{tabular}{clcccc}
\hline Código & Localidad & Temperatura ${ }^{\circ} \mathbf{C}$ & $\mathbf{p H}$ & Conductividad $\boldsymbol{\mu S} / \mathbf{c m}$ & Oxígeno $\%$ \\
\hline 1 & Conso 1 & 4.8 & 7.6 & 12.6 & - \\
2 & Conso 2 & - & 6.3 & 12.0 & 75.4 \\
3 & Pradoalbar & 15.4 & 6,7 & 19.6 & 77.8 \\
4 & Cenza & - & 7.1 & 20.2 & 79.1 \\
5 & Cernado & 15.4 & 6.5 & 15.9 & 80.3 \\
6 & Palleiras & 12.9 & 6.5 & 12.3 & 87.5 \\
7 & Caneiro & 16.7 & 6.6 & 65.1 & 95.0 \\
8 & Tornos & 12.5 & 6.8 & 13.1 & 89.4 \\
9 & Grixoa & 17.0 & 6.4 & 12.2 & 81.7 \\
10 & Fonte Ribeira Pequena & 11.9 & 5.8 & 28.3 & 81.5 \\
\hline
\end{tabular}



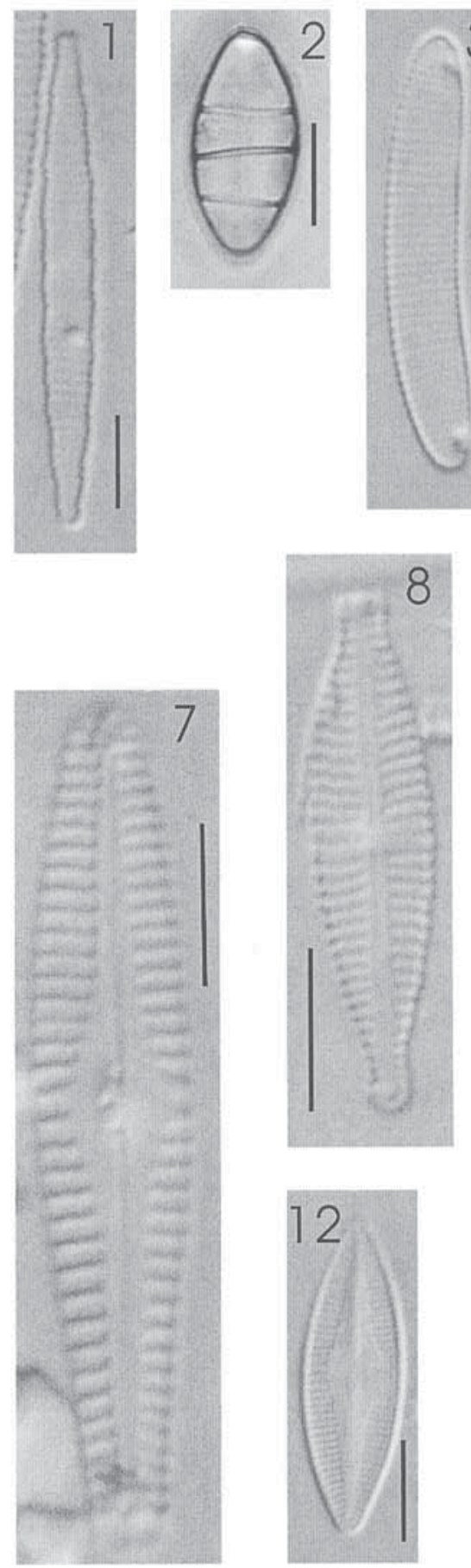
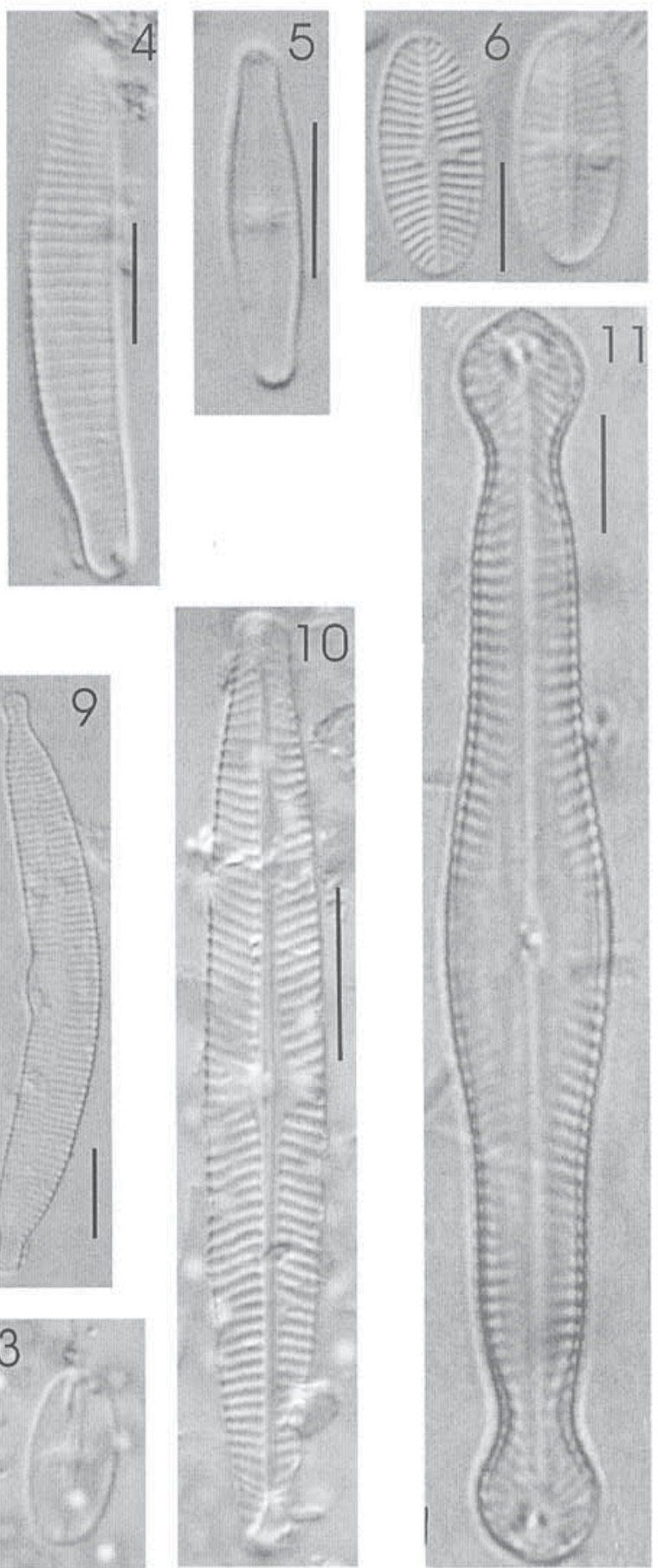

Figura 2. 1. Fragilaria capucina, 2. Diatoma mesodon, 3. Eunotia intermedia, 4. Eunotia minor, 5. Achnanthidium minutissimum, 6. Planothidium distinctum, 7. Gomphonema rhombicum, 8. G. parvulum, 9. Hannaea arcus, 10. Navicula angusta, 11. Pinnularia polyonca, 12. Nupela lapidosa, 13. Psammothidium cf. subatomoides. Escala: $6 \mu \mathrm{m}$. 1. Fragilaria capucina, 2. Diatoma mesodon, 3. Eunotia intermedia, 4. Eunotia minor, 5. Achnanthidium minutissimum, 6. Planothidium distinctum, 7. Gomphonema rhombicum, 8. G. parvulum, 9. Hannaea arcus, 10. Navicula angusta, 11. Pinnularia polyonca, 12. Nupela lapidosa, 13. Psammothidium $c f$. subatomoides. scale: $6 \mu \mathrm{m}$ 
Tabla 3. Índices diatomológicos: IPS, IBD y CEE y porcentaje de las especies utilizadas en cada muestra para su cálculo. IPS, IBD, and CEE diatom indexes, and percentage of the species used in each sample for its calculation.

\begin{tabular}{clcccccc}
\hline Código & Localidad & IPS & \% & IBD & \% & CEE & \% \\
\hline 1 & Conso 1 & 19.6 & 96.3 & 18.5 & 74.07 & 18.3 & 66.67 \\
2 & Conso 2 & 19.1 & 100 & 18.4 & 60 & 17.9 & 80 \\
3 & Pradoalbar & 19.9 & 100 & 20 & 80 & 19.2 & 90 \\
4 & Cenza & 19.4 & 100 & 19 & 91.3 & 18.9 & 78.26 \\
5 & Cernado & 18.5 & 100 & 18.5 & 72.73 & 15.6 & 77.27 \\
6 & Palleiras & 19.0 & 100 & 19.2 & 66.67 & 17.9 & 66.67 \\
7 & Caneiro & 17.2 & 100 & 15.1 & 66.67 & 14.3 & 66.67 \\
8 & Tornos & 19.4 & 100 & 20 & 100 & 16.8 & 100 \\
9 & Grixoa & 17.7 & 100 & 17.1 & 62.54 & 14.3 & 65.38 \\
10 & Fonte Ribeira Pequena & 17.7 & 100 & 19.5 & 70 & 13.4 & 66.67 \\
\hline
\end{tabular}

típica de aguas poco mineralizadas, oscilando entre 12 y $65.1 \mu \mathrm{S} / \mathrm{cm}$ y el porcentaje de oxígeno varía entre 75.4 y $95 \%$.

Se han identificado un total de 65 taxones: 2 a nivel de género, 60 a nivel de especie y 3 a nivel de variedad, siendo las especies más abundantes (Fig. 2), Achnanthidium minutissimum (21\%), Diatoma mesodon (15\%), Fragilaria capucina (5.5\%), Gomphonema parvulum (4.5\%), G. rhombicum (8\%) y Hannaea arcus $(6.5 \%)$.

Las especies más frecuentes (presentes en más de 6 localidades) son Achnanthidium minutissimum, Diatoma mesodon, Eunotia minor, Fragilaria capucina, Gomphonema parvulum, $G$. rhombicum, Hannaea arcus, Navicula angusta y Pinnularia subcapitata.

La aplicación de los índices de calidad IPS, IBD y CEE dio como resultado la siguiente clasificación de las aguas: calidad muy buena, valores superiores a 17 , y buena, valores entre 13 y 17 (Tabla 3). Cinco de las diez localidades estudiadas: Cenza, Conso 1 y 2, Pradoalbar y Fonte Ribeira Pequena, muestran valores que indican una calidad muy buena para los tres índices aquí estudiados. Comparando los tres índices, el IPS es con el que se obtienen valores más altos, así todos los puntos tendrían una calidad del agua muy buena. El IBD da valores de calidad de agua muy buena para todas las localidades excepto una, Caneiro. Por último, el CEE es el que da los peores resultados ya que clasifica a cinco de las localidades: Caneiro, Cernado, Grixoa, Palleiras y Tornos, en calidad buena.

\section{DISCUSIÓN}

Se observa que las comunidades de diatomeas del MCG se distribuyen dependiendo del sustrato de la cuenca. Así tenemos que en las localidades con sustrato metamórfico abundan las siguientes diatomeas: Achnanthidium minutissimum, Gomphonema rhombicum, Hannaea arcus, Navicula angusta y Surirella linearis. En las localidades con sustrato sedimentario aparecen como muy abundantes: A. minutissimum, Eunotia intermedia, Fragilaria capucina, Karayevia oblongella, Pinularia subcapitata, Psammothidium cf. subatomoides y Tabellaria floculosa. Y en las localidades con sustrato granítico hay una mayor abundancia de: A. minutissimum, F. capucina, Nitzschia dissipata (aparece sólo en Caneiro, que tiene influencia antrópica), $P$. cf. subatomoides, $S$. linearis y $T$. floculosa. Las aguas en estos ríos con sustrato granítico están ligeramente más oxigenadas y tienen una menor conductividad que el resto de los ríos. A. minutissimum, aparece en los tres tipos de cuenca, pero es menos abundante en las graníticas, $S$. linearis también aparecen en los tres tipos de cuenca pero con menor abundancia en las sedimentarias. Las cuencas sedimentarias y graníticas presentan más especies comunes.

La mayoría de las diatomeas encontradas tienen una distribución cosmopolita, sin embargo, Eunotia intermedia, G. rhombicum, K. oblongella, Navicula angusta, Nupela lapidosa, Planothidium distinctum y Pinnularia polyonca son es- 
pecies típicas de ríos de montaña (Krammer et Lange-Bertalot, 1986, 1988,1991a, b).

De las especies más abundantes, Diatoma mesodon, H. arcus y G. rhombicum son indicadoras de aguas de calidad muy buena y A. minutissimum de aguas de calidad buena (Ector, 1992; Prygiel \& Coste, 2000).

La localidad que tiene los peores resultados en cuanto a los valores de los índices es Caneiro, que presenta el máximo valor de la conductividad $(65.1 \mu \mathrm{S} / \mathrm{cm})$ pues se trata de una zona del río acondicionada como playa fluvial, siendo por tanto la localidad con más influencia humana dentro de las estudiadas. A pesar de esto ni los valores de los índices ni el valor de la conductividad indican que sea una zona con contaminación.

En cuanto al porcentaje de especies utilizadas para el cálculo de cada índice, se observa que es el IPS el que más taxones tiene en cuenta (>96.3\%). Ector (1992) y De la Peña (2003) ya indicaron que se trataba del índice más adecuado para los ríos de Galicia.

\section{CONCLUSIONES}

Este trabajo contribuye a ampliar el conocimiento de la flora diatomológica en Galicia, obteniéndose un catálogo de 65 taxones. A pesar de caracterizarse la comunidad gallega por poseer un gran número de ríos, apenas existen trabajos publicados sobre este tema. Es por ello que se han encontrado en el Macizo Central Gallego, 2 nuevas citas para España, 3 para Galicia y 17 para la provincia de Ourense (que serán objeto de otra publicación más extensa y detallada).

Con el estudio del estado de la calidad de las aguas mediante índices que utilizan las comunidades de diatomeas perifíticas, se obtienen valores que oscilan entre 13.4 y 20 , por tanto las localidades estudiadas en el Macizo Central Gallego presentan aguas de buena a muy buena calidad. El índice IPS es el que da mejores resultados para los ríos gallegos estudiados hasta el momento.

\section{AGRADECIMIENTOS}

Agradecemos a Sergi Sabater y a su equipo, de la Universidad de Girona, el haber facilitado la identificación de algunas especies y el asesoramiento en la metodología para llevar a cabo este trabajo. Este estudio es una contribución al proyecto PGIDT01MAM20001 financiado por la Xunta de Galicia.

\section{REFERENCIAS}

ABOAL M., M. ALVÁREZ COBELAS, J. CAMBRA \& L. ECTOR. 2003. Floristic list of the non marine diatoms (Bacillariophyceae) of Iberian Peninsula, Balearic Islands and Canary Islands. Update taxonomy and bibliography. En: Diatom Monographs. A. Witkowski (ed.). Vol. 4. Berlín, ARG Gantner Verlag K.G. 639 pp.

BOURRELLY, P. 1968. Les Algues d'eau douce. Initiation a la systématique, II: Les Algues jaunes et brunes, Chrysophycées, Phéophycées, Xanthophycées et Diatomées. París. Éditions N. Boubée. 438 pp.

CARBALLEIRA A., C. DEVESA, R. RETUERTO, E. SANTILLÁN y F. UCIEDA. 1983. Bioclimatología de Galicia. A Coruña: Fundación Pedro Barrie de La Maza. 391 pp.

DE LA PEÑA, S. 2003. Biomonitorización de la calidad del agua de ríos de la provincia de A Coruña usando diatomeas. Tesis de Licenciatura. Universidad de A Coruña. $117 \mathrm{pp}$.

ECTOR, L. 1992. Control de la calidad biológica de las aguas superficiales en la red de aforos de Galicia-costa mediante diatomeas bénticas. En: Calidad del agua en las estaciones de aforo de los ríos de Galicia. Años hidrológicos 1989-90, 1990-91. Fundación Empresa Universidad Gallega (FEUGA). 76-157. Consellería de Ordenación do Territorio e Obras Públicas, Xunta de Galicia (ed.). Santiago de Compostela.

EUROPEAN COMMITTEE FOR STANDARDIZATION, 2002. Water quality-Guidance standard for the routine sampling and pretreatment of benthic diatoms from rivers. prEN 13946. 14 pp.

GAMUNDI, J. 1911. Diatomeas de Santiago de Compostela y sus alrededores. Boletín de la Real Sociedad Española de Historia Natural, 11:388394. 
GERMAIN, H. 1981. Flore des diatomées. París. Société Nouvelle des Éditions Boubée. 444 pp.

HUSTEDT, F. 1930. Die Süsswasser-Flora Mitteleuropas. Heft 10: Bacillariophyta (Diatomaceae). Koenigstein. Otto Koeltz Science Publhishers. 466 pp.

I.G.M.E. 1977. Mapa Geológico de España 1: 50000. Hoja 227 (Manzaneda). Madrid: Servicio de Publicaciones de Ministerio de Industria y Energía.

KELly, M. G., A. CAZAUBON, E. CORING, A. DELL-UOMO, L. ECTOR, B. GOLDSMITH, H. GUASCH, J. HÜRLIMANN, A. JARLMAN, B. KAWECKA, J. KWANDRANS, R. LAUGASTE, E. A. LINDSTR ØM, M. LEITAO, P. MARVAN, J. PADISÁK, E. PIPP, J. PRYGIEL, E. ROTT, S. SABATER, H. DAM \& J. VIZINET. 1998. Recommendations for routine sampling of diatoms for water quality assessments in Europe. J. Appl. Phycol., 10: 215-224.

KRAMMER, K. 2000. The genus Pinnularia. En: Diatoms of Europe. Diatoms of the European Inland Waters and Comparable habitats. H. Lange Bertalot (ed.). Vol 1. Koenigstein. ARG Gantner Verlag K.G. 703 pp.

KRAMMER, K. 2002. Cymbella. En: Diatoms of Europe. Diatoms of the European Inland Waters and Comparable habitats. H. Lange Bertalot (ed.). Vol 3. Koenigstein. ARG Gantner Verlag K.G. 584 pp.

KRAMMER, K. 2003. Cymbopleura, Delicata, Navicymbula, Gomphocymbellopsis, Afrocymbella. En: Diatoms of Europe. Diatoms of the European Inland Waters and Comparable Habitats. $\mathrm{H}$. Lange-Bertalot (ed.). Vol. 4: Koenigstein. A. R. G. Gantner Verlag K.G, 530 pp.

KRAMMER, K. \& H. LANGE-BERTALOT. 1986. Bacillariophyceae 1 Teil: Naviculaceae. En: Die Süßwasserflora von Mitteleuropa. H. Ettl, J. Gärtner, H. Heynig \& D. Mollenhauer (eds.). Stuttgart. Gustav Fisher Verlag. 876 pp.

KRAMMER, K. \& H. LANGE-BERTALOT. 1988. Bacillariophyceae 2 Teil Bacillariaceae, Epithemiaceae, Surirellaceae. En: Die Süßwasserflora von Mitteleuropa. H. Ettl, J. Gärtner, H. Heynig \& D. Mollenhauer (eds.). Stuttgart. Gustav Fisher Verlag. 596 pp.

KRAMMER, K. \& H. LANGE-BERTALOT. 1991a. Bacillariophyceae 3 Teil: Centrales, Fragilariaceae, Eunotiaceae. En: Die Süßwasserflora von Mitteleuropa. H. Ettl, J. Gärtner, H. Heynig \& D.
Mollenhauer (eds.). Stuttgart. Gustav Fisher Verlag. 575 pp.

KRAMMER, K. \& H. LANGE-BERTALOT. $1991 b$. Bacillariophyceae 4 Teil: Achnanthaceae, Kritische Ergäzungen zu Navicula (Lineolatae) und Gomphonema. En: Die Süßwasserflora von Mitteleuropa. H. Ettl, J. Gärtner, H. Heynig \& D. Mollenhauer (eds.). Stuttgart. Gustav Fisher Verlag. 876 pp.

LANGE-BERTALOT, H. 2001. Navicula sensu stricto. 10 Genera separated from Navicula sensu lato Frustulia. En: Diatoms of Europe. Diatoms of the European Inland Waters and Comparable habitats. H. Lange-Bertalot (ed.). Vol 2. Koenigstein. ARG Gantner Verlag K.G. 526 pp.

LANGE-BERTALOT, H. \& K. KRAMER. 1989. Achnanthes, eine Monographie der Gattung. En: Bibliotheca Diatomologica. H. Lange-Bertalot (ed.). Band 18. Berlin. J. Cramer. 393 pp.

LÓPEZ RODRÍGUEZ, $\mathrm{M}^{\mathrm{a}} \mathrm{C}$. y $\mathrm{M}^{\mathrm{a}}$ PENALTA RODRÍGUEZ. 2004. Aportación al conocimiento de la flora ficológica del Macizo Central Gallego (N.O. España). Anales de Biología de Murcia, 26: 79-91.

MARGALEF, R. 1955. Comunidades bióticas de las aguas dulces del noroeste de España. Publicaciones del Instituto de Biología Aplicada, 21: 5-85.

MARGALEF, R. 1956. Algas de agua dulce del norte de España. Publicaciones del Instituto de Biología Aplicada, 22: 5-47.

NOGUEROL, A. 1993. Algas dulceacuícolas de la Sierra de Invernadeiro (Orense, N.O. España). Nova Acta Científica Compostelana (Bioloxía) 4: 5-13.

PENALTA RODRÍGUEZ, $\mathrm{M}^{\mathrm{a}}$ et $\mathrm{M}^{\mathrm{a}} \mathrm{C}$. LÓPEZ RODRÍGUEZ, 2006. Contribution à la flore des diatomées épilithiques du Massif Central Galicien (Ourense, Espagne). Simbioses, 14: 21-25.

PRYGIEL, J. \& M. COSTE. 2000. Guide méthodologique pour la mise en oeuvre de l'Indice Biologique Diatomées. NF T 90-354. Agence de l'eau Artois-Picardie, Cemagref Bordeaux, Douai, $134 \mathrm{pp}$.

PRYGIEL, J., B. A. WHITTON \& J. BUKOWSKA. 1999. Use of Algae for Monitoring Rivers III. Proceedings of the 3rd European Workshop, Douai, 1997. Agence de l'Eau Artois-Picardie, Douai. 271 pp.

VARELA, M. 1976. Diatomeas de Santiago de Compostela y alrededores. Tesis de Licenciatura. Universidade de Santiago de Compostela. 95 pp. 
VARELA, M. 1982. Adiciones a la flora de diatomeas de agua dulce de Galicia. Collectanea botanica, 13(2): 977-985.

VARELA, M., B. RODRÍGUEZ y E. COSTAS.
1992. Inventario de diatomeas de agua doce de Galicia. Cadernos da Àrea de Ciencias Biológicas. (Inventarios do Seminario de Estudos Galegos), Vol IX: 11-55. 